\title{
Tendon cells isolated from patients with persistent shoulder tendinopathy show dysregulated resolution responses
}

Rotator cuff tendinopathy is a common cause of pain and disability and a substantial social and economic burden affecting athletic and aging individuals. ${ }^{1}$ Patients refractory to physical therapy have increasingly been treated with arthroscopic sub-acromial decompression (ASAD), although the benefit to patients has recently been challenged by evidence from randomized placebo-controlled surgical trials. ${ }^{2}$ The mechanisms governing whether inflammation persists or resolves in patients with supraspinatus tendinopathy in the absence of a tendon tear are poorly understood. We previously identified increased expression of genes associated with resolving inflammation and tissue repair in tissue biopsies collected from pain-free compared to persistently painful patients after ASAD. ${ }^{3}$ However, the biological mechanisms governing persistent pain and inflammation in patient's refractory to treatment remain unidentified. To gain insights into the biological processes underpinning resolution of shoulder tendinopathy, we investigated the bioactive lipid mediator (LM) profiles of tendon stromal cells isolated from patients with supraspinatus tendinopathy to determine whether LM profiles differed between cells isolated from symptomatic and asymptomatic patients before and after ASAD. We hypothesized that tendon stromal cells isolated from painful post-treatment patients would show dysregulated resolution responses.

Tissue biopsies were collected from symptomatic patients $(\mathrm{n}=5)$ during general anesthesia prior to ASAD (painful pre-treatment group). Samples were also collected from patients between 2 and 4 years after ASAD in whom pain had resolved completely (pain-free post-treatment, $n=5$ ) or pain persisted (painful post-treatment, $n=4$ ). All patients were aged between 38 and 65 years and had not received cortisone treatment for 3 months prior to inclusion in the study. Patient age, gender, and BMI did not differ significantly between cohorts. In post-treatment patients, tendon biopsies were performed under ultrasound guidance and local anesthesia using a validated biopsy technique. ${ }^{4}$ Tissues were collected under research ethics from South Central Oxford (14/SC/0222). Full informed consent according to the Declaration of Helsinki was obtained from all patients.
Tendon stromal cells $\left(\mathrm{CD} 45^{-} \mathrm{CD} 34^{-}\right.$fibroblasts) were isolated from biopsy samples using previously published protocols. ${ }^{3,5}$ Passage 1-2 cells were cultured to confluence in the presence/absence of IL-1 $\beta\left(10 \mathrm{ng} \mathrm{mL}^{-1}\right)$ in DMEMF12 phenol red-free medium, containing $1 \%$ heat-inactivated human serum and $1 \%$ penicillin-streptomycin. IL-1 $\beta$ was selected as this proinflammatory cytokine is known to induce expression of NF-kB target genes highly expressed in supraspinatus tendinopathy. ${ }^{3}$ Cells were incubated for 24 hours until harvest of the media and lysate for LM profiling, which was performed using a previously described protocol. ${ }^{6}$ RNA was isolated from collected lysates and RT-qPCR performed as previously described, ${ }^{3}$ to determine whether 15-PGDH mRNA expression varied between tendon stromal cells isolated from the above patient cohorts.

Tendon stromal cells derived from painful pre-treatment, painful post-treatment, and pain-free post-treatment patients showed distinct clustering of the LM profiles (Figure 1A, 1B). Cells from painful post-treatment patients showed increased cumulative levels of specialized proresolving mediators (SPMs) compared to respective cells from painful pre-treatment and pain-free post-treatment patients (Figure 1C, 1D). Cumulative SPM from cells isolated from painful post-treatment patients was increased compared to respective cells from pain-free posttreatment patients under IL-1 $\beta$-stimulated conditions $(P=0.03)$. Assessment of individual LM concentrations demonstrated significant increases in specific SPMs including maresin 2 and resolvin $\mathrm{D} 5_{\mathrm{n} 3 \mathrm{DPA}}$ in cells isolated from the tendons of post-treatment compared to pre-treatment patients (Figure $1 \mathrm{E}$ and F). In cell incubations from painful post-treatment patients, we also found increased levels of inflammation-initiating eicosanoids including $\mathrm{PGE}_{2}$ and $\mathrm{PGF}_{2 \alpha}$ (Figure 1G). Tendon cells isolated from pain-free post-treatment showed reduced 15-PGDH mRNA expression compared to respective cells from painful pre-treatment patients $(P=0.03$, Figure $1 \mathrm{H})$.

This study presents a unique opportunity to understand the mechanisms underpinning persistent tendon pain and inflammation. Increased levels of SPM and inflammation-initiating eicosanoids in cells from persistently painful patients suggest that the concentrations of SPM are not sufficient to

This is an open access article under the terms of the Creative Commons Attribution License, which permits use, distribution and reproduction in any medium, provided the original work is properly cited.

(C) 2019 The Authors. Translational Sports Medicine Published by John Wiley \& Sons Ltd 


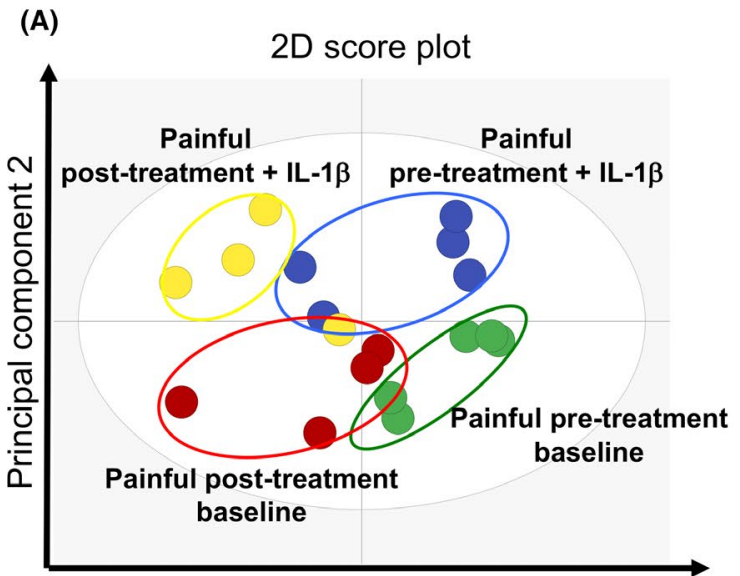

Principal component 1

(B)

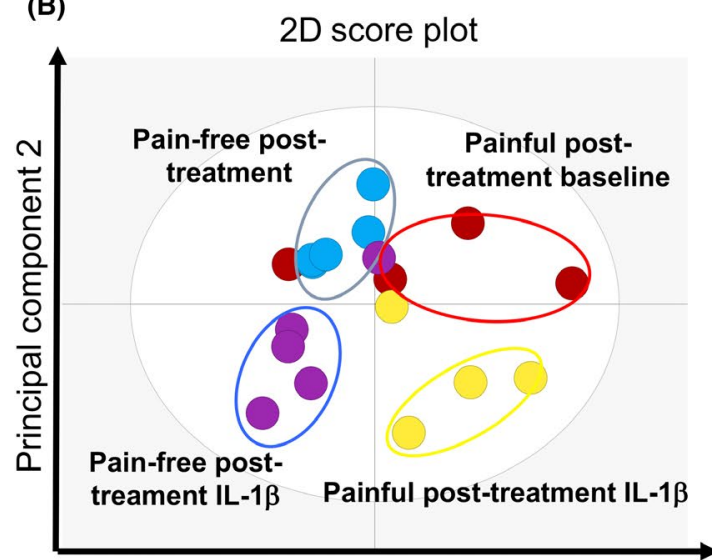

Principal component 1

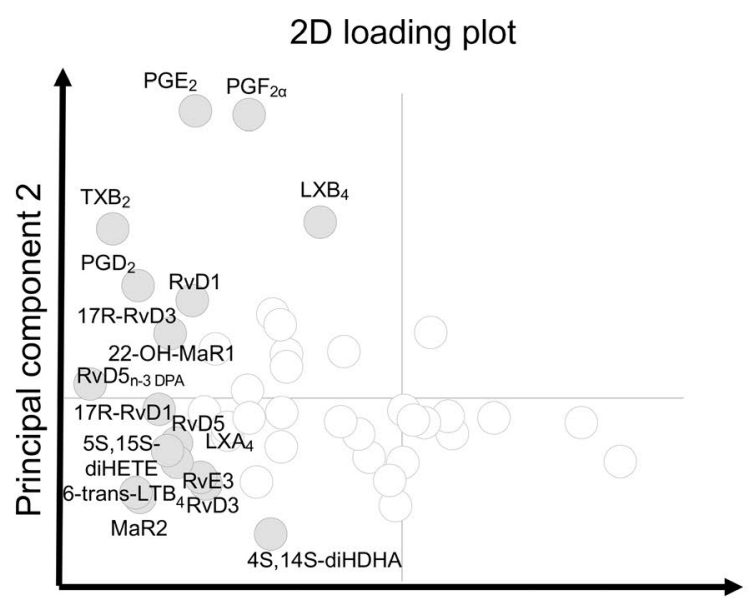

Principal component 1

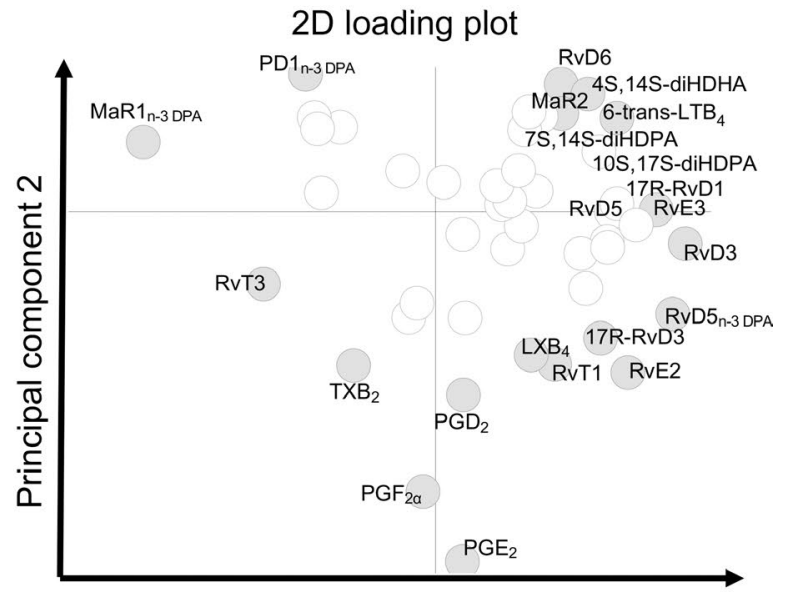

Principal component 1
(C)

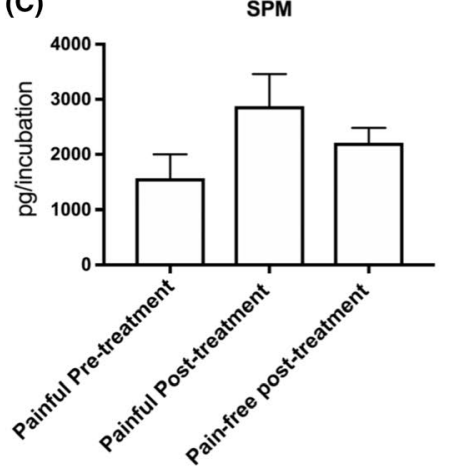

(F)

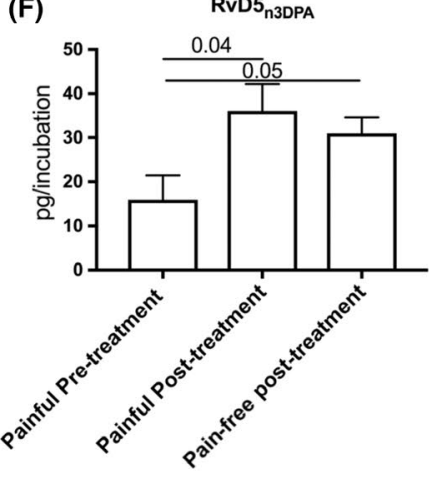

(D)

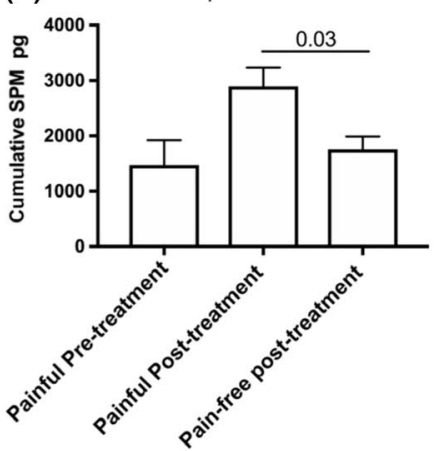

(G)

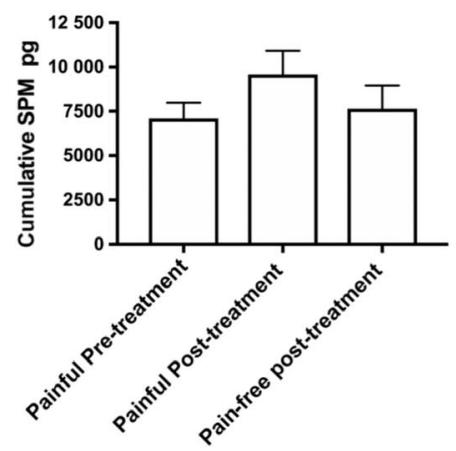

(E)
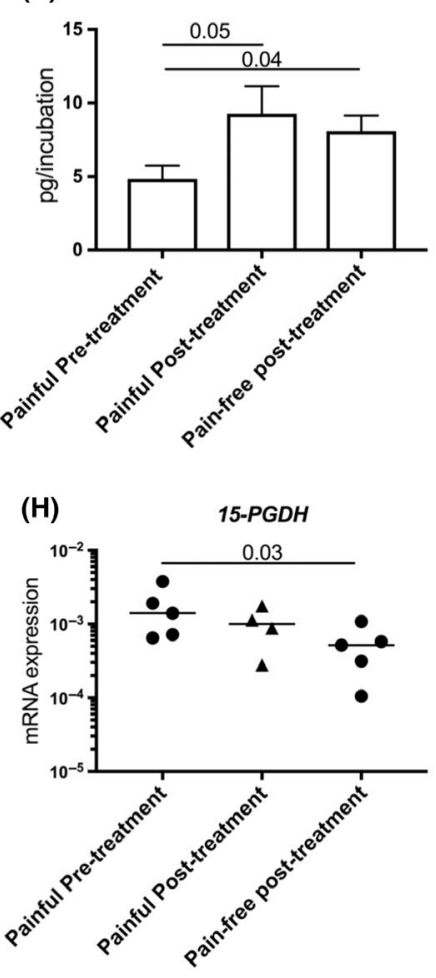
F I G U R E 1 Resolution responses of tendon stromal cells isolated from painful and pain-free patients before and after treatment. Tendon stromal cells were derived from painful pre-treatment $(n=5)$, painful post-treatment $(n=4)$, and pain-free post-treatment patients $(n=5)$ and cultured in the presence or absence of IL-1 $\beta\left(10 \mathrm{ng} \mathrm{mL}^{-1}\right)$ for $24 \mathrm{~h}$. Media and cells were harvested, and bioactive lipid mediators (LM) were extracted and profiled. (A, B) 2-dimensional score plots showing partial least square discriminant analysis together with associated score plots that highlight differences in LM profiles between patient cohorts and the effects of IL-1 $\beta$ treatment. Cumulative levels of specialized proresolving mediators (SPMs) in these incubations are shown under (C) baseline unstimulated and (D) IL-1 $\beta$-stimulated conditions. (E, F) Concentrations of maresin 2 and RvD5 $5_{\text {n3DPA }}$ found to be differentially regulated between patient tendon stromal cell incubations under baseline unstimulated conditions. (G) Cumulative levels of inflammation-initiating eicosanoids (IIE) in IL-1 $\beta$-stimulated patient tendon stromal cell incubations, results are shown as means and SEM. (H) 15-PGDH mRNA expression in patient tendon stromal cell incubations. Gene expression is normalized to $\beta$-actin, and bars show median values

counter-regulate ongoing inflammatory processes in cells from these patients, reminiscent of a dysregulated resolution response characteristic of chronic inflammation. We previously identified stromal cells isolated from patients with supraspinatus tendon tears also showed dysregulated resolution responses compared to respective cells isolated from healthy volunteer tendons. ${ }^{6}$ In shoulder tendon tears, dysregulated resolution responses were attributed to 15-PGDH mediated further metabolism and inactivation of SPM. In the current study, we identified reduced $15-P G D H$ in tendon cells isolated from pain-free post-treatment compared to respective cells from painful pre-treatment patients. It was not possible to longitudinally match patient samples in this study cohort. However, our findings suggest an association exists between normalization of the proinflammatory/proresolving axis within tendon-derived stromal cells and the resolution of patient symptoms. Collectively, these findings provide new insights into the disease mechanisms underpinning shoulder tendon disease, demonstrating that stromal cells isolated from patients with persistent shoulder tendinopathy show dysregulated resolution responses. New therapeutic approaches to treat shoulder tendinopathy are required that target tendon stromal cells and promote resolution of tendon inflammation.

\section{ACKNOWLEDGEMENTS}

SGD is funded by an Oxford-UCB Prize Fellowship in Biomedical Sciences. Research at Oxford University is supported by the NIHR Oxford Biomedical Research Centre. The views expressed are those of the authors and not necessarily the NHS or the Department of Health. JD received funding from the European Research Council (ERC) under the European Union's Horizon 2020 research and innovation program (grant no: 677542) and the Barts Charity (grant no: MGU0343). JD is also supported by a Sir Henry Dale Fellowship jointly funded by the Wellcome Trust and the Royal Society (grant 107613/Z/15/Z). We thank the patient participants who agreed to provide tissue samples.

\section{Key message}

Tendon cells from patients with persistent shoulder tendinopathy show dysregulated resolution responses.

\section{CONFLICT OF INTEREST}

The authors declare no conflicts of interest exist.

$$
\begin{array}{r}
\text { Stephanie G. Dakin }{ }^{1} \text { ID } \\
\text { Romain A. Colas }{ }^{2} \\
\text { Benjamin J. F. Dean }{ }^{1} \\
\text { Kim Wheway }{ }^{1} \\
\text { Bridget Watkins }{ }^{1} \\
\text { Jesmond Dalli }^{2,3} \\
\text { Andrew J. Carr }
\end{array}
$$

${ }^{1}$ Botnar Research Centre, Nuffield Orthopaedic Centre, Nuffield Department of Orthopaedics, Rheumatology and Musculoskeletal Sciences, University of Oxford, Oxford, UK

${ }^{2}$ Lipid Mediator Unit, William Harvey Research

Institute, Barts and The London School of Medicine and Dentistry, Queen Mary University of London, London, UK

${ }^{3}$ Centre for Inflammation and Therapeutic

Innovation, Queen Mary University of London, London, UK

Email:stephanie.dakin@ndorms.ox.ac.uk

Dalli and Carr are equal senior author contribution.

\section{ORCID}

Stephanie G. Dakin (D) https://orcid.org/0000-0003-2470-1031

\section{REFERENCES}

1. Mitchell C, Adebajo A, Hay E, Carr A. Shoulder pain: diagnosis and management in primary care. BMJ. 2005;331(7525):1124-1128.

2. Beard DJ, Rees JL, Cook JA, et al. Arthroscopic subacromial decompression for subacromial shoulder pain (CSAW): a multicentre, 
pragmatic, parallel group, placebo-controlled, three-group, randomised surgical trial. Lancet. 2018;391(10118):329-338.

3. Dakin SG, Martinez FO, Yapp C, et al. Inflammation activation and resolution in human tendon disease. Sci Transl Med. 2015;7(311):311ra173.

4. Murphy RJ, Floyd Dean BJ, Wheway K, Watkins B, Morrey ME, Carr AJ. A novel minimally invasive ultrasound-guided technique to biopsy supraspinatus tendon. Oper Tech Orthop. 2013;23(2):56-62.
5. Dakin SG, Buckley CD, Al-Mossawi MH, et al. Persistent stromal fibroblast activation is present in chronic tendinopathy. Arthritis Res Ther. 2017;19(1):16.

6. Dakin SG, Ly L, Colas RA, et al. Increased 15-PGDH expression leads to dysregulated resolution responses in stromal cells from patients with chronic tendinopathy. Sci Rep. 2017;7(1):11009. 